\title{
HISTOPLASMOSE PULMONAR AGUDA: RELATO DE UMA MICROEPIDEMIA*
}

\author{
Erick Malheiro Leoncio Martins ${ }^{1}$, Edson Marchiori ${ }^{2}$, Simone Duarte Damato ${ }^{1}$, \\ Aline Serfaty Pozes ${ }^{1}$, Ana Carina Gamboa da Silva ${ }^{3}$, Marcos Dalston ${ }^{4}$
}

Resumo Os autores relatam uma microepidemia de histoplasmose pulmonar, com cinco crianças que desenvolveram a doença em um período de 7 a 14 dias após a limpeza de um forno desativado para produção de carvão vegetal. Todas apresentaram quadro de febre alta persistente, tosse seca, astenia e anorexia, com 28 dias de evolução. Quando buscaram atendimento médico, uma delas encontrava-se taquipnéica, febril, com hepatomegalia e palidez cutânea, estando as restantes em regular estado geral e já sem febre. As radiografias de tórax demonstravam, em todos os casos, infiltrados reticulonodulares grosseiros, difusos e bilaterais, além de linfonodomegalias hilares. As tomografias computadorizadas evidenciaram pequenos nódulos difusos, com distribuição aleatória, além das linfonodomegalias. Os diagnósticos foram confirmados por meio da imunodifusão em gel para Histoplasma capsulatum, que foi positiva em todas as amostras pareadas coletadas com 15 dias de intervalo. Apenas uma criança necessitou de internação, por causa de importante queda no estado geral, sendo realizado tratamento de suporte e observação. Todas as crianças evoluíram com melhora clínica, sem o uso de antifúngicos, e foram submetidas a tomografias de controle após cerca de 50 dias, que demonstraram importante regressão das lesões.

Unitermos: Radiologia; Histoplasmose pulmonar; Microepidemia.

Abstract Acute pulmonary histoplasmosis: report of an outbreak.

The authors report a pulmonary histoplasmosis outbreak occurring in five children after the cleaning of a deactivated coal furnace. The symptoms were high fever, dry cough, asthenia and anorexia. By the time parents seeked for medical care, only one child remained symptomatic with fever, tachypnea and hepatomegaly. All the patients had similar radiological findings. Chest films showed diffuse, bilateral, reticulonodular infiltrates and lymph node enlargement. Computed tomography showed small nodules with a random distribution and hilar and mediastinal lymph nodes. Diagnosis was confirmed by positive gel immunodiffusion for Histoplasma capsulatum. Only one patient needed to be hospitalized to receive supportive treatment. The use of antifungal agents was not necessary in any of the patients. After $\mathbf{5 0}$ days all patients were submitted to computed tomography studies that revealed significant remission of the lesions.

Key words: Radiology; Pulmonary histoplasmosis; Outbreak.

\section{INTRODUÇÃO}

A histoplasmose pulmonar é uma infecção geralmente associada com exposição a fezes de pássaros, que são um bom meio de cultura para o Histoplasma capsulatum, permitindo sua propagação e viabilidade por vários $\operatorname{anos}^{(\mathbf{1})}$.

* Trabalho realizado no Departamento de Radiologia e no Serviço de Doenças Infecciosas e Parasitárias da Universidade Federal Fluminense (UFF), Niterói, RJ, e no Serviço de Radiodiagnóstico do Hospital Universitário Clementino Fraga Filho da Universidade Federal do Rio de Janeiro (UFRJ), Rio de Janeiro, RJ.

1. Médicos Residentes de Radiologia da UFF.

2. Professor Titular de Radiologia da UFF, Coordenador Adjunto do Curso de Pós-Graduação em Radiologia da UFRJ.

3. Médica do Serviço de Radiologia da UFF.

4. Professor Adjunto do Serviço de Doenças Infecciosas e Parasitárias da UFF.

Endereço para correspondência: Prof. Dr. Edson Marchiori. Rua Thomaz Cameron, 438, Valparaíso. Petrópolis, RJ, 25685-120. E-mail: edmarchiori@zipmail.com.br

Recebido para publicação em 27/9/2002. Aceito, após revisão, em 11/11/2002.
As microepidemias ocorrem quando um número pequeno de indivíduos acha-se em uma região restrita e com solo contaminado, sendo expostos aos esporos dos fungos presentes em suspensão aérea ${ }^{(2)}$. Os locais e as circunstâncias que ocasionam surtos epidêmicos são variados, destacando-se, entre outras situações, visitas a grutas, limpeza de galinheiros ${ }^{(3)}$, construção de pontes e torres ${ }^{(4)}$ e cortes de árvores caídas ${ }^{(\mathbf{1})}$.

As alterações radiológicas mais comuns são áreas pequenas e esparsas de pneumonite, com infiltrados alveolar e intersticial, quase sempre associados a linfonodomegalias hilar e/ou mediastinal ${ }^{(2)}$. As tomografias computadorizadas geralmente evidenciam pequenos nódulos de distribuição aleatória, que representam a disseminação miliar para ambos os pulmões.

Neste trabalho são apresentados cinco casos de histoplasmose pulmonar ocorri- dos em crianças, após limpeza de forno desativado para produção de carvão vegetal.

As radiografias convencionais demonstraram, em todos os pacientes, infiltrados reticulonodulares grosseiros, difusos e bilaterais, além de linfonodomegalias hilares. As tomografias computadorizadas de tórax evidenciaram pequenos nódulos difusos, de distribuição aleatória, com linfonodomegalias.

\section{CASUÍSTICA E MÉTODO}

Um grupo de cinco crianças, com idades entre 4 e 11 anos, foi encaminhado ao Serviço de Doenças Infecciosas e Parasitárias da Universidade Federal Fluminense, Niterói, RJ, apresentando quadro de febre alta persistente, tosse seca, astenia e anorexia, com 28 dias de evolução. Todas eram de uma mesma família, sendo três meninas 
e dois meninos. Cerca de 30 dias antes do início da doença, as crianças haviam descoberto um forno desativado para a produção de carvão vegetal, no interior do qual passaram a realizar seus folguedos. O forno encontrava-se escavado em um barranco, possuía apenas uma entrada e era habitado por morcegos. Poderia ser considerado como uma caverna artificial. Entre 7 e 14 dias antes do início da doença, o local foi inteiramente limpo e varrido pelas crianças.

Ao exame físico, uma delas, um menino de quatro anos de idade, apresentava palidez cutânea, febre $\left(38^{\circ} \mathrm{C}\right)$, taquipnéia, estertores subcrepitantes em ambas as bases pulmonares e hepatomegalia. Não havia linfonodomegalias em seu exame físico. As demais crianças apresentavam-se em regular estado geral e já afebris. Observaram-se linfonodomegalias cervicais em uma delas e hepatomegalia em outras duas.

As radiografias de tórax evidenciaram, em todos os casos, infiltrados reticulonodulares grosseiros, difusos e bilaterais, além de linfonodomegalias hilares. As tomografias computadorizadas de tórax demonstraram pequenos nódulos difusos, com distribuição aleatória. Em todos os casos a imunodifusão em gel para $H$. capsulatum, realizada na Fundação Instituto Oswaldo Cruz, Rio de Janeiro, RJ, foi positiva em amostras pareadas coletadas com 15 dias de intervalo.

Apenas um dos pacientes, o menino de quatro anos de idade, necessitou de internação por causa da importante queda no estado geral, sendo realizado apenas tratamento de suporte e observação. Todos os casos evoluíram com melhora clínica, sem o uso de antifúngicos, e tomografias de controle realizadas após cerca de 50 dias demonstraram regressão das lesões.

Foram realizadas tomografias computadorizadas de tórax em todos os pacientes, e fotografados com janelas adequadas para avaliação do parênquima pulmonar e do mediastino. Todos os exames foram complementados com técnica de alta resolução, que consistiu em cortes seqüenciais com colimação fina (1 a $2 \mathrm{~mm}$ ), a intervalos de 10 a $20 \mathrm{~mm}$, e com algoritmo de reconstrução nítida (“ósseo”).

\section{RESULTADOS}

Neste trabalho foram analisadas as tomografias computadorizadas de cinco pacientes com histoplasmose pulmonar aguda, sendo que três eram do sexo feminino (60\%). A idade variou entre 4 e 11 anos (média de 8 anos).

As alterações tomográficas observadas foram a presença de pequenos nódulos difusos, com distribuição aleatória, associados a linfonodomegalias mediastinais e hilares bilaterais (100\% dos pacientes). Não houve predomínio das lesões em nenhuma região pulmonar específica (Figuras 1 e 3).

Cerca de 50 dias após as primeiras tomografias, foram realizados exames de controle que demonstraram regressão importante das lesões parenquimatosas e discreta redução das linfonodomegalias (Figuras 2 e 4 ).

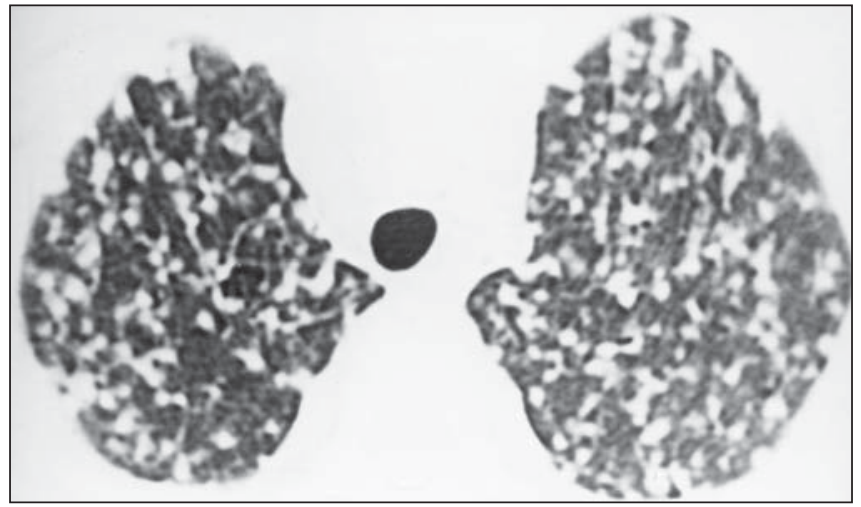

A

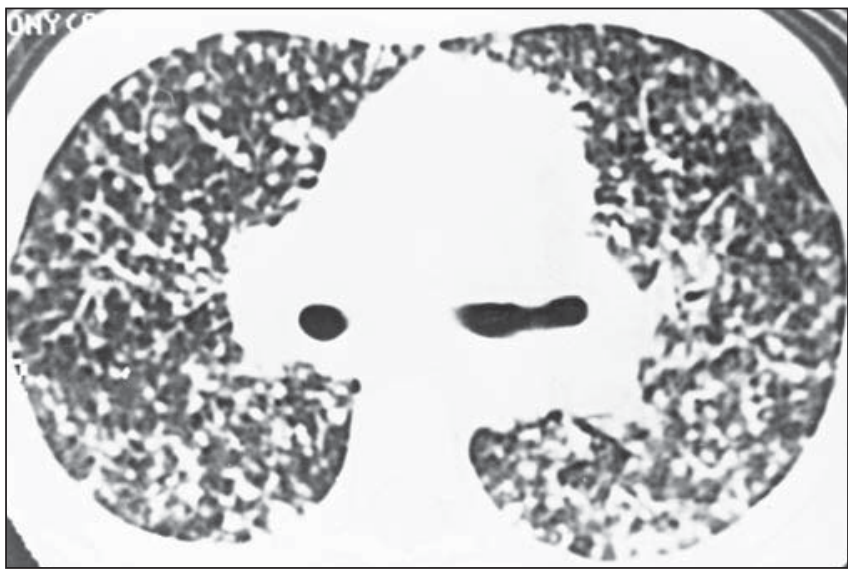

B
Figura 1. Cortes de tomografia computadorizada de alta resolução, em três diferentes níveis, demonstrando pequenos nódulos com distribuição aleatória, dispersos de maneira homogênea pelos pulmões. Observam-se, ainda, algumas áreas de confluência nas regiões posteriores dos lobos inferiores e linfonodomegalias hilares bilaterais.

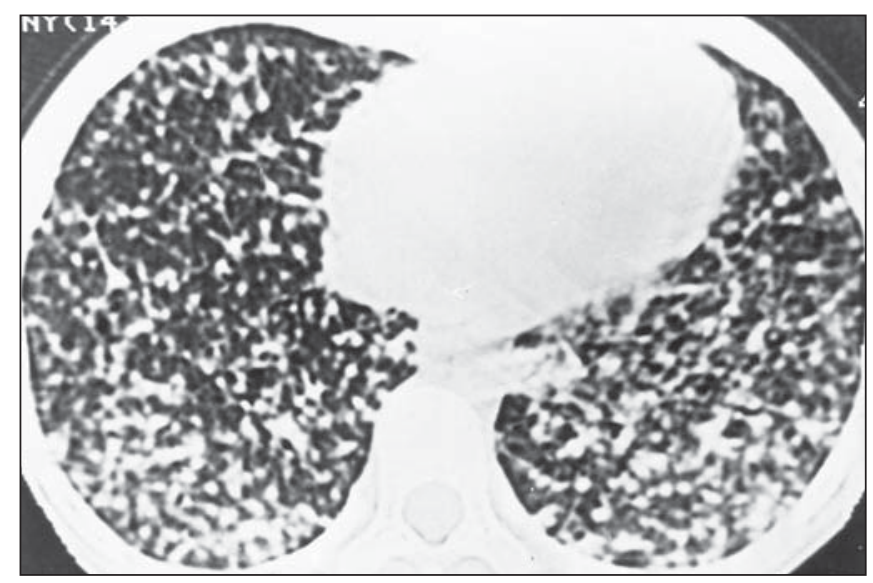

C 


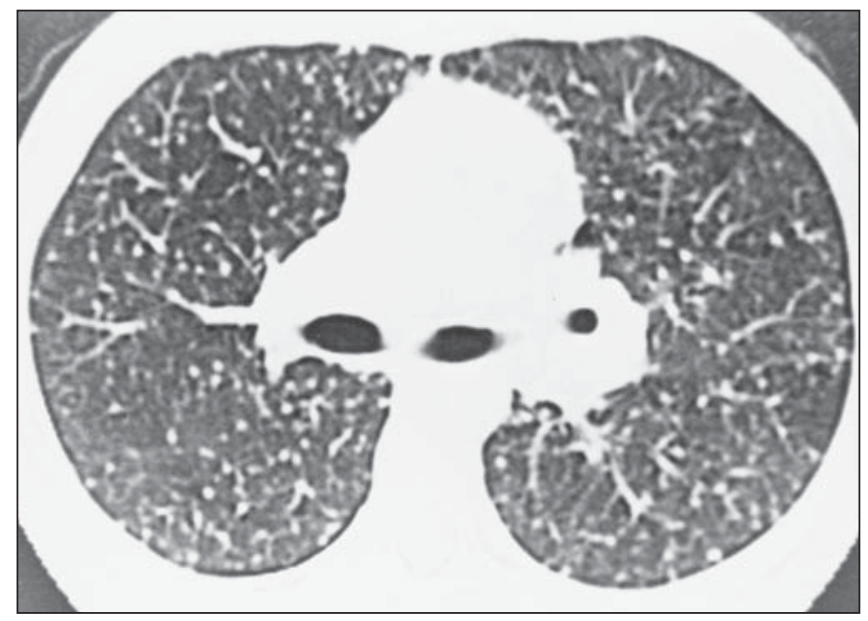

A

Figura 2. Controle, após 50 dias, do paciente da Figura 1 evidenciando regressão importante das lesões nodulares e discreta involução das massas linfonodais.

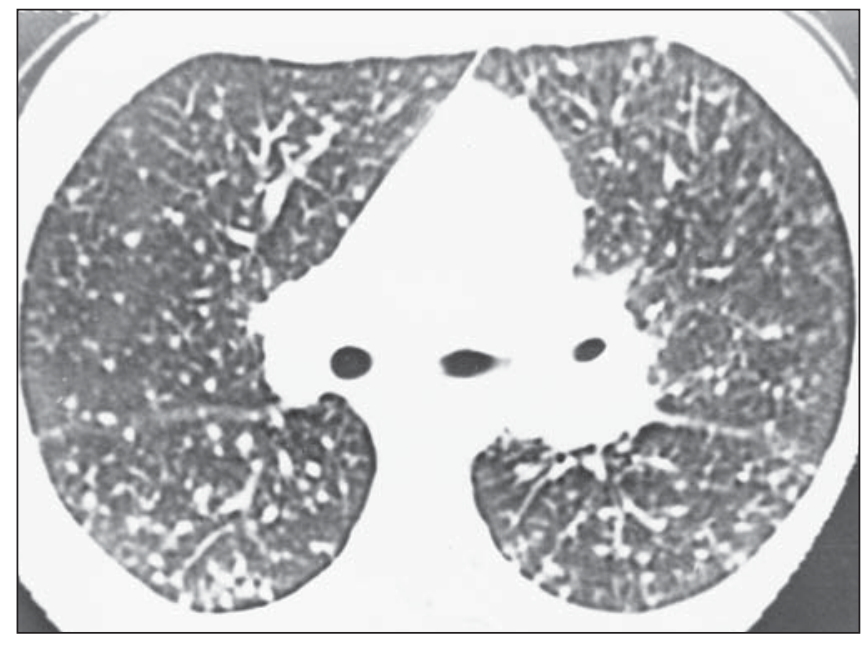

A

Figura 3. Cortes de tomografia computadorizada de alta resolução dos campos médios e inferiores dos pulmões evidenciando pequenos nódulos de distribuição randômica. Nota-se ainda linfonodomegalia hilar bilateral.

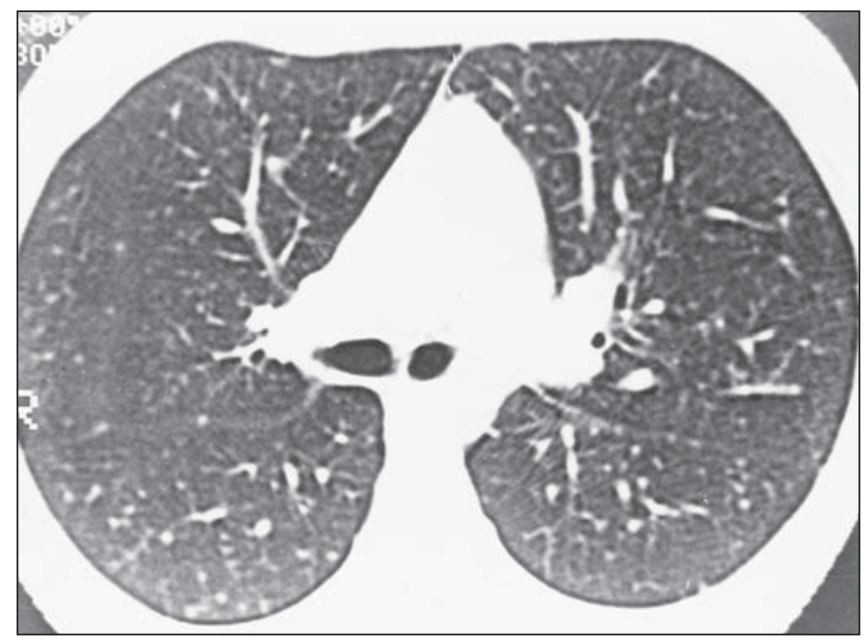

A

Figura 4. Mesmo paciente da Figura 3, examinado 50 dias após. Cortes em planos semelhantes aos da Figura 3, nos quais se observam melhora evolutiva das lesões parenquimatosas e involução das linfonodomegalias hilares.
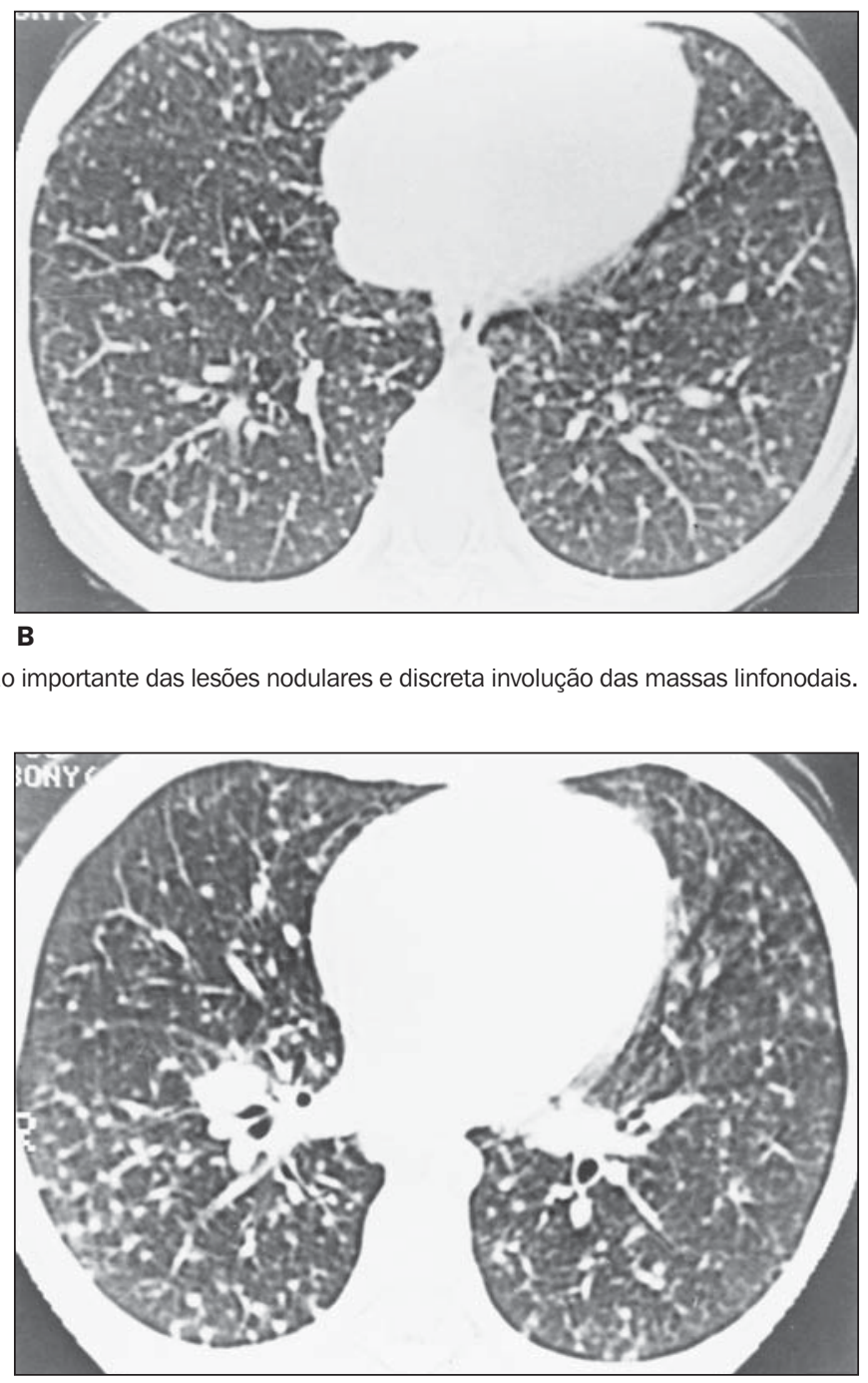

B

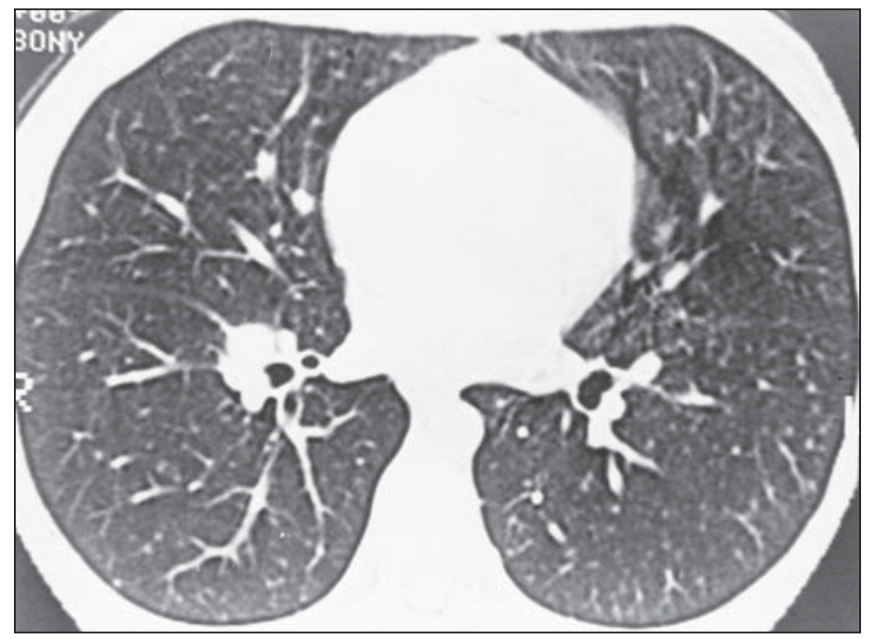

B 


\section{DISCUSSÃO}

O H. capsulatum é um fungo dimórfico, cosmopolita, geralmente encontrado nos solos na sua forma saprófita ou filamentosa ${ }^{(5)}$. Quando incubado a $37^{\circ} \mathrm{C}$ assume a forma parasitária, que consiste em elementos leveduriformes arredondados ou ovalados.

Existe uma relação definida entre a histoplasmose e a presença de aves. Os pássaros não hospedam o fungo, pois a sua alta temperatura corporal não permite seu crescimento. Entretanto, o solo enriquecido por fezes permite o crescimento desses organismos. Os morcegos, como são mamíferos, podem hospedar o histoplasma em seu trato digestivo, disseminando-o a partir do seu habitat, que consiste de cavernas, porões e buracos em árvores ${ }^{(\mathbf{6})}$.

Uma vez estabelecido no solo, o fungo persiste indefinidamente, mesmo que este local seja abandonado pelos pássaros. No solo contaminado, a maior parte das partículas infectantes é encontrada na camada mais superficial, e a sua propagação se faz principalmente pelo vento ${ }^{(7)}$.

Um dos aspectos mais curiosos da histoplasmose é a descrição das microepidemias $^{(3)}$. Os surtos epidêmicos decorrem da movimentação de agrupamentos humanos em pequenos ambientes com solo rico em fungos, favorecendo a dispersão aérea das suas partículas e a contaminação simultânea dos indivíduos ${ }^{(2)}$. Os locais e as circunstâncias que ocasionam essas microepidemias são bastante variados e incluem cortes de árvores caídas, manipulação de ocos de árvores ${ }^{(\mathbf{1})}$, visitas a grutas, construção de pontes e torres, demolição de casas $\operatorname{antigas}^{(2)}$, limpeza ou terraplanagem em locais de pernoite de pássaros, como parques e sítios $^{(2)}$, limpeza de galinheiros e asilos abandonados, pintura de pontes metálicas $^{(3)}$, exploração de túmulos no antigo Egito $^{(3)}$, etc.

Uma das maiores epidemias de histoplasmose ocorreu em uma pequena cidade de Indianápolis, EUA, após o desmatamento de um bosque. $\mathrm{O}$ vento levou a poeira criada aos prédios vizinhos, resultando em um saldo de 120.000 pessoas presumivelmente infectadas, 488 casos da doença e 60 pacientes com histoplasmose fatal ou muito grave $\mathrm{e}^{(\mathbf{8})}$. Outro interessante surto coletivo ocorreu perto de Nashville, EUA, em 1977. Após a derrubada de um centenário carvalho, o proprietário convocou 42 amigos, que em festivo piquenique se encarregaram de transformar a árvore caída em toras. Após cerca de 14 dias, 48\% dos participantes apresentaram histoplasmose aguda, sendo que três tiveram que ser hospitalizados. Dois cachorros também adoeceram com lesões pulmonares ${ }^{(\mathbf{9})}$.

No Brasil, a doença existe de norte a sul do país. Porém, a observação de várias microepidemias nos Estados do Rio de Janeiro e do Rio Grande do Sul, tanto em áreas rurais como urbanas, coloca estas regiões como áreas de alta endemicidade ${ }^{(3,10)}$.

A infecção pelo $H$. capsulatum geralmente ocorre quando os pequenos esporos (micronídeas), trazidos pelo vento, são inalados e chegam aos alvéolos pulmonares $^{(11)}$. Outras vias de infecção, como, por exemplo, pele e trato gastrintestinal, são $\operatorname{raras}^{(\mathbf{6})}$. Na temperatura corporal, os fungos sofrem germinação, liberando formas leveduriformes que serão fagocitadas pelos macrófagos. No interior dos macrófagos as leveduras não são destruídas e se multiplicam, resultando em broncopneumonia que envolve lóbulos pulmonares secundários contíguos. Por via linfática, os parasitas chegam aos linfonodos regionais, onde produzem novo foco inflamatório, configurando, assim, o complexo pulmonar e linfonodal primário. Posteriormente, há disseminação dos organismos por via hematogênica, sendo o baço um órgão freqüentemente acometido.

Nos indivíduos imunocompetentes a imunidade celular específica, desencadeada por linfócitos T, se desenvolve em um período de uma a quatro semanas, sendo manifestada por testes cutâneos com resultados positivos $^{(\mathbf{6}, 11)}$. O desenvolvimento da hipersensibilidade leva a uma intensa reação inflamatória, com necrose caseosa e controle da infecção. Há melhora clínica espontânea e as lesões começam a involuir ou calcificar ${ }^{(6)}$.

Conforme a quantidade de elementos infectantes inalados, a infecção primária ou a reinfecção pode transcorrer sem sintomas (exposição leve) ou com sintomas tanto mais graves quanto maior a carga infectante (exposição moderada ou intensa). Infecções assintomáticas e uma grande variedade de síndromes autolimitadas são comuns na histoplasmose. Após exposições leves, cerca de $1 \%$ dos pacientes apresenta doença sintomática e autolimitada; os 99\% restantes apresentam infecção não reconhecida clinicamente. A maior parte dos indivíduos sintomáticos tem história de exposição a locais altamente contaminados, como, por exemplo, limpeza de porão e galinheiros, e desenvolvem doença pulmonar com febre, tosse, cefaléia, dor torácica e fadiga. Em geral, há melhora sem tratamento $^{(2,3)}$.

A infecção aguda pode ainda levar a outras manifestações da doença, como, por exemplo, as formas cavitária crônica e a disseminada $^{(2,11,12)}$.

A infecção disseminada é a forma mais grave da histoplasmose, geralmente ocorre em indivíduos com comprometimento da imunidade celular e é fatal se não tratada. Sua gravidade é variável e depende da idade do paciente, órgãos atingidos e extensão das lesões ${ }^{(4,7)}$. É caracterizada por doença progressiva com evidências de acometimento extrapulmonar. A maioria dos pacientes apresenta febre prolongada, emagrecimento, hepatoesplenomegalia, lesões cutâneas e mucosas, meningite ou cerebrite, insuficiência supra-renal, pancitopenia e alterações nas provas de função hepática. As radiografias de tórax são normais em um terço dos pacientes, mas podem demonstrar infiltrados miliares ou difusos em $35 \%$, infiltrados focais em $20 \%$ e/ou linfonodopatia em $20 \%$. O diagnóstico pode ser feito pelo isolamento dos fungos em culturas e amostras de tecidos extrapulmonares, ou por meio da detecção de antígenos ${ }^{(11)}$

Em indivíduos com doenças pulmonares concomitantes, como enfisema pulmonar, há o risco de desenvolvimento da forma cavitária crônica da doença, que consiste em uma infecção granulomatosa fibrocavitária progressiva $^{(\mathbf{4 , 5}, 7)}$, que se caracteriza por tosse produtiva, sudorese, emagrecimento, fadiga e infiltrados pulmonares apicais fibróticos com escavações. Nas fases iniciais pode se manifestar como uma pneumonite intersticial que evolui com fibrose e escavação. Sem tratamento, pode 
haver extensão da doença para ambos os pulmões, formação de novas cavidades, aumento das preexistentes e surgimento de fístulas broncopleurais ${ }^{(\mathbf{1 1})}$.

Uma das formas da histoplasmose que não responde à terapia é a mediastinite fibrosante, que se manifesta por compressão de estruturas mediastinais ${ }^{(\mathbf{1 1})}$. As radiografias de tórax podem demonstrar alargamento mediastinal ou linfonodomegalia hilar calcificada. A tomografia computadorizada é mais útil na demonstração das alterações fibróticas ${ }^{(\mathbf{1}, 13)}$.

$\mathrm{Na}$ histoplasmose aguda as alterações radiográficas são geralmente inespecíficas, sendo mais comum a visualização de áreas únicas ou múltiplas de consolidação. Linfonodopatias hilares e mediastinais também são freqüentemente observadas, mas o derrame pleural é raro ${ }^{(\mathbf{1 4})}$. Em alguns indivíduos, especialmente em crianças, o envolvimento dos linfonodos pode ser mais proeminente que as alterações pulmonares. A infecção aguda pode ainda levar a seqüelas tardias, como granulomas e fibrose mediastinal $^{(6)}$.

Após grandes exposições, a maioria dos pacientes desenvolve doença pulmonar sintomática e grave, sendo em alguns casos disseminada. Na maior parte dos casos as radiografias demonstram infiltrados miliares, que consistem em pequenas opacidades nodulares de 3 a $4 \mathrm{~mm}$, difusas, e que após cerca de dois a oito meses tendem a calcificar ou sofrer fibrose. Estes pacientes geralmente apresentam insuficiência respiratória causada por extenso comprometimento pulmonar. Podem ainda ser encontradas extensas áreas de consolidações, não segmentares e bilaterais, sendo a resolução das alterações radiográficas lenta.

Severo et al. ${ }^{(\mathbf{1 0 )}}$ estudaram 19 casos de histoplasmose pulmonar aguda, sendo $63 \%$ homens, com idades que variavam de 8 a 59 anos. Dezoito pacientes apresentaram quadro agudo de febre, emagrecimento, tosse e sudorese noturna, associado a infiltrado micronodular difuso. Linfonodomegalias bilaterais estavam presentes nas radiografias de três desses pacientes.

Em nosso trabalho todas as crianças apresentavam infiltrado intersticial nodular difuso, sendo as linfonodomegalias pouco expressivas nas radiografias. Entretanto, apesar do extenso comprometimento pulmonar, os sintomas dos pacientes eram pouco expressivos, e apenas um paciente necessitou de internação. As radiografias de controle realizadas após o tratamento demonstraram rápida melhora das lesões.

Quanto às alterações observadas nas tomografias computadorizadas de alta resolução, nossos resultados foram bastante semelhantes aos de Gharib e Stern ${ }^{(\mathbf{1 3})}$. Esses autores citam que os nódulos têm distribuição aleatória, sendo o aspecto bastante semelhante ao da tuberculose miliar. Vale ressaltar que poucos trabalhos na literatura descreveram os aspectos tomográficos da histoplasmose pulmonar aguda.

\section{REFERÊNCIAS}

1. Pladson TR, Stiles MA, Kuritsky JN. Pulmonary histoplasmosis. A possible risk in people who cut decayed wood. Chest 1984;86:435-8.

2. Wanke B, Capone D. O pulmão na histoplasmose. Arq Bras Med 1990;64:381-8.

3. Paula A, Aidé MA. As microepidemias de histoplasmose do Estado do Rio de Janeiro. J Bras Med 1985;49:18-28.

4. Jones TF, Swinger GL, Craig AS, McNeil MM, Kaufman L, Schaffner W. Acute pulmonary histoplasmosis in bridge workers: a persistent problem. Am J Med 1999;106:480-2.

5. Bradsher RW. Histoplasmosis and blastomycosis. Clin Infect Dis 1996;22 Suppl 2:S102-11.

6. Wheat J. Histoplasmosis: recognition and treatment. Clin Infect Dis 1994;19 Suppl 1:S19-27.

7. Straus SE, Jacobson ES. The spectrum of histoplasmosis in a general hospital: a review of 55 cases diagnosed at Barnes Hospital between 1966 and 1977. Am J Med Sci 1980;279:147-58.

8. Wheat LJ, Slama TG, Norton JA, et al. Risk factors for disseminated or fatal histoplasmosis: analysis of a large urban outbreak. Ann Intern Med 1982;96: 159-63.

9. Ward JI, Weeks M, Allen D, et al. Acute histoplasmosis: clinical, epidemiologic and serologic findings of an outbreak associated with exposure to a fallen tree. Am J Med 1979;66:587-95.

10. Severo LC, Oliveira FM, Irion K, Porto NS, Londero AT. Histoplasmosis in Rio Grande do Sul, Brazil: a 21-year experience. Rev Inst Med Trop São Paulo 2001;43:183-7.

11. Prager RL, Burney DP, Waterhouse G, Bender HW Jr. Pulmonary, mediastinal, and cardiac presentations of histoplasmosis. Ann Thorac Surg 1980;30: 385-90.

12. Gurney JW, Conces DJ Jr. Pulmonary histoplasmosis. Radiology 1996;199:297-306.

13. Gharib AM, Stern EJ. Radiology of pneumonia. Med Clin North Am 2001;85:1461-91.

14. Goldman M, Johnson PC, Sarosi GA. Fungal pneumonias. The endemic mycoses. Clin Chest Med 1999;20:507-19. 